\title{
Mean Field Theory for Lossy Nonlinear Composites
}

\author{
K. W. Yu \\ Department of Physics, The Chinese University of Hong Kong, \\ Shatin, New Territories, Hong Kong
}

\begin{abstract}
The mean-field theory for lossy nonlinear composites, described by complex and field-dependent dielectric functions, is presented. By using the spectral representation of linear composites with identical microstructure, we develop self-consistent equations for the effective response. We examine two types of microstructure, namely, the Maxwell-Garnett approximation and the effective medium approximation to illustrate the theory.
\end{abstract}

PACS Numbers: 72.20.Ht, 64.60.Ak, 72.60.+g 


\section{INTRODUCTION}

Recently, we proposed a mean-field theory (MFT) for nonlinear composites [1]. The theory was applied to nonlinear transport properties of conducting composites at zero frequency, described by real conductivities and provided good agreement with numerical simulation [1]. However, for dielectric materials near relaxation, the loss may be important. It is tempting to extend the MFT to complex and nonlinear permitivities. The local constitutive relation of the composite system is given by

$$
\mathbf{D}=\chi\left(\mathbf{E}^{*} \cdot \mathbf{E}\right) \mathbf{E}
$$

where $\chi=\chi(\mathbf{x})$ is the (position dependent) complex third-order nonlinear coefficient. In what follows, we denote $|\mathbf{E}|^{2}=\mathbf{E}^{*} \cdot \mathbf{E}$ and $\mathbf{E}^{2}=\mathbf{E} \cdot \mathbf{E}$ for convenience. The effective response is defined as the volume average of $\mathbf{D}$

$$
\chi_{e} \mathbf{E}_{0}^{2} \mathbf{E}_{0}=\frac{1}{V} \int_{V} d V \mathbf{D}(\mathbf{x}),
$$

where $V$ is the volume of composites and $\mathbf{E}_{0}$ is the applied (average) field and is taken to be real:

$$
\mathbf{E}_{0}=\frac{1}{V} \int_{V} d V \mathbf{E}(\mathbf{x})
$$

The corresponding boundary-value problem of nonlinear composite media consists of the field equations:

$$
\begin{aligned}
& \nabla \cdot \mathbf{D}=0, \\
& \nabla \times \mathbf{E}=0 .
\end{aligned}
$$

There exists a potential $\varphi$ so that $\mathbf{E}=\nabla \varphi$. The boundary condition for $\varphi$ is

$$
\varphi=\varphi_{0}=\mathbf{E}_{0} \cdot \mathbf{x}
$$

on surface $(S)$ of the composite and $\varphi_{0}$ is real. Consider the integral $(1 / V) \int_{V} d V \mathbf{D} \cdot \mathbf{E}$. Through the field equations, we can convert it into an integral over the surface $(S)$ of the 
composite: $(1 / V) \int_{S} d \mathbf{S} \cdot(\mathbf{D} \varphi)$, which is equal to $(1 / V) \int_{S} d \mathbf{S} \cdot\left(\mathbf{D} \varphi_{0}\right)$ because $\varphi=\varphi_{0}$ on $S$. Converting the surface integral back to the volume integral, we obtain:

$$
\frac{1}{V} \int_{V} d V \mathbf{D} \cdot \mathbf{E}=\frac{1}{V} \int_{V} d V \mathbf{D} \cdot \mathbf{E}_{0}
$$

Hence we find an equivalent expression for the effective response:

$$
\chi_{e} \mathbf{E}_{0}^{4}=\frac{1}{V} \int_{V} d V \mathbf{D} \cdot \mathbf{E} .
$$

Let us consider a lossy nonlinear composite in which inclusions of complex nonlinear coefficient $\chi_{1}$, present at volume fraction $p_{1}$, are randomly embedded in a host medium of $\chi_{2}$, present at volume fraction $p_{2}$, with the application of an external uniform field $\mathbf{E}_{0}$. Note that $p_{1}+p_{2}=1$. From Eqs.(11) and (8), we have therefore an expression for the effective response:

$$
\chi_{e} \mathbf{E}_{0}^{4}=p_{1} \chi_{1}\left\langle\left|\mathbf{E}_{1}\right|^{2} \mathbf{E}_{1}^{2}\right\rangle+p_{2} \chi_{2}\left\langle\left|\mathbf{E}_{2}\right|^{2} \mathbf{E}_{2}^{2}\right\rangle,
$$

where

$$
\left\langle\left|\mathbf{E}_{i}\right|^{2} \mathbf{E}_{i}^{2}\right\rangle=\frac{1}{V_{i}} \int_{V_{i}} d V|\mathbf{E}|^{2} \mathbf{E}^{2}, \quad i=1,2
$$

denotes the local field average within the $i$ th component. Similar to the derivation of Eq.(可), as $\varphi^{*}=\varphi_{0}$ on $S$, we arrive at

$$
\frac{1}{V} \int_{V} d V \mathbf{D} \cdot \mathbf{E}^{*}=\frac{1}{V} \int_{V} d V \mathbf{D} \cdot \mathbf{E}_{0}
$$

which implies an alternative expression for the effective response:

$$
\chi_{e} \mathbf{E}_{0}^{4}=p_{1} \chi_{1}\left\langle\left(\left|\mathbf{E}_{1}\right|^{2}\right)^{2}\right\rangle+p_{2} \chi_{2}\left\langle\left(\left|\mathbf{E}_{2}\right|^{2}\right)^{2}\right\rangle
$$

where

$$
\left\langle\left(\left|\mathbf{E}_{i}\right|^{2}\right)^{2}\right\rangle=\frac{1}{V_{i}} \int_{V_{i}} d V\left(|\mathbf{E}|^{2}\right)^{2}, \quad i=1,2
$$

We should remark that the different types of local field averages defined in Eqs.([10) and ([13) are generally not equal to each other. It is interesting to note that they both give the same 
effective response [Eqs.(9) and (12)]. However, the nonlinear partial differential equations pertaining to the boundary-value problem cannot be solved analytically. We shall invoke the mean-field theory [1] to obtain an approximate expression for the effective response. According to previous work [1], we consider the linear constitutive relation:

$$
\mathbf{D}=\epsilon \mathbf{E},
$$

where $\epsilon=\epsilon(\mathbf{x})$ is the (position dependent) complex dielectric constant. By definition, we find the effective linear response:

$$
\epsilon_{e} \mathbf{E}_{0}=\frac{1}{V} \int_{V} d V \epsilon \mathbf{E}
$$

From Eq.(7), we have therefore

$$
\epsilon_{e} \mathbf{E}_{0}^{2}=p_{1} \epsilon_{1}\left\langle\mathbf{E}_{1}^{2}\right\rangle+p_{2} \epsilon_{2}\left\langle\mathbf{E}_{2}^{2}\right\rangle
$$

From Eq.(11), we have alternatively

$$
\epsilon_{e} \mathbf{E}_{0}^{2}=p_{1} \epsilon_{1}\left\langle\left|\mathbf{E}_{1}\right|^{2}\right\rangle+p_{2} \epsilon_{2}\left\langle\left|\mathbf{E}_{2}\right|^{2}\right\rangle
$$

This expression will be useful for calculating $\left\langle\left|\mathbf{E}_{i}\right|^{2}\right\rangle$. In order to estimate $\chi_{e}$, we invoke the decoupling approximation [2]

$$
\left\langle\left|\mathbf{E}_{i}\right|^{2} \mathbf{E}_{i}^{2}\right\rangle=\left\langle\left|\mathbf{E}_{i}\right|^{2}\right\rangle\left\langle\mathbf{E}_{i}^{2}\right\rangle, \quad i=1,2 .
$$

within the $i$ th component. This assumption is good in microstructure for which the local electric field is nearly uniform within the $i$ th component, but less accurate when these fluctuations are large, as in a random composite near the percolation threshold [2]. With this assumption,

$$
\chi_{e} \mathbf{E}_{0}^{4}=p_{1} \chi_{1}\left\langle\left|\mathbf{E}_{1}\right|^{2}\right\rangle\left\langle\mathbf{E}_{1}^{2}\right\rangle+p_{2} \chi_{2}\left\langle\left|\mathbf{E}_{2}\right|^{2}\right\rangle\left\langle\mathbf{E}_{2}^{2}\right\rangle
$$

Comparing Eqs.(16) and (19), it is tempting to write $\epsilon_{1}=\chi_{1}\left\langle\left|\mathbf{E}_{1}\right|^{2}\right\rangle, \epsilon_{2}=\chi_{2}\left\langle\left|\mathbf{E}_{2}\right|^{2}\right\rangle$ and $\epsilon_{e}=\chi_{e} \mathbf{E}_{0}^{2}$. Hence, within the decoupling approximation, we may interpret the nonlinear 
component as a linear dielectric material with a field-dependent dielectric constant. Suppose $\epsilon_{e}$ is known as a function of its constituent dielectric constants,

$$
\epsilon_{e}=F\left(\epsilon_{1}, \epsilon_{2}, p_{1}\right)
$$

then we find the local field averages [1]

$$
\begin{aligned}
& p_{1}\left\langle\mathbf{E}_{1}^{2}\right\rangle=\frac{\partial \epsilon_{e}}{\partial \epsilon_{1}} \mathbf{E}_{0}^{2} \\
& p_{2}\left\langle\mathbf{E}_{2}^{2}\right\rangle=\frac{\partial \epsilon_{e}}{\partial \epsilon_{2}} \mathbf{E}_{0}^{2}
\end{aligned}
$$

However, we have to determine $\left\langle\left|\mathbf{E}_{i}\right|^{2}\right\rangle$ in the $i$ th component, which is generally not equal to $\left\langle\mathbf{E}_{i}^{2}\right\rangle$. Thus a straightforward application of the previous formalism [1] is impossible. We resort to an alternative approach based on the spectral representation [3].

In what follows, we shall consider two important types of microstructures: (1) Dispersion microstructures as in the Maxwell-Garnett approximation [四] and (2) symmetric microstructures as in the Bruggeman effective medium approximation [5].

\section{MAXWELL-GARNETT APPROXIMATION}

The Maxwell-Garnett approximation (MGA) is good for dispersion microstructures and the theory is inherently non-symmetrical [4]. For convenience, we consider the case in which component 1 is embedded in component 2. For a linear composite of $\epsilon_{1}$ and $\epsilon_{2}$ at volume fractions $p_{1}$ and $p_{2}$ respectively, the MGA reads [4]:

$$
\frac{\epsilon_{e}-\epsilon_{2}}{\epsilon_{e}+(d-1) \epsilon_{2}}=p_{1} \frac{\epsilon_{1}-\epsilon_{2}}{\epsilon_{1}+(d-1) \epsilon_{2}}
$$

where $d$ is the dimensionality of composites. Solving Eq.(23), we obtain

$$
\epsilon_{e}=\frac{\epsilon_{2}\left[\epsilon_{1}\left(d p_{1}+p_{2}\right)+\epsilon_{2}(d-1) p_{2}\right]}{p_{2} \epsilon_{1}+\left(d-p_{2}\right) \epsilon_{2}} .
$$

From Eqs.(21) and (22), we can calculate the local field averages:

$$
p_{1}\left\langle\mathbf{E}_{1}^{2}\right\rangle=\frac{p_{1} d^{2} \epsilon_{2}^{2}}{\left[p_{2} \epsilon_{1}+\left(d-p_{2}\right) \epsilon_{2}\right]^{2}} \mathbf{E}_{0}^{2} .
$$




$$
p_{2}\left\langle\mathbf{E}_{2}^{2}\right\rangle=\left(1-\frac{p_{1} d\left[d \epsilon_{2}^{2}-p_{2}\left(\epsilon_{1}-\epsilon_{2}\right)^{2}\right]}{\left[p_{2} \epsilon_{1}+\left(d-p_{2}\right) \epsilon_{2}\right]^{2}}\right) \mathbf{E}_{0}^{2} .
$$

In MGA, the local field in the inclusion is uniform, we can determine $\mathbf{E}_{1}$ and hence $\mathbf{E}_{1}^{*}$ explicitly, we find

$$
p_{1}\left\langle\left|\mathbf{E}_{1}\right|^{2}\right\rangle=\frac{p_{1} d^{2}\left|\epsilon_{2}\right|^{2}}{\left|p_{2} \epsilon_{1}+\left(d-p_{2}\right) \epsilon_{2}\right|^{2}} \mathbf{E}_{0}^{2}
$$

From Eq.(17) and using Eqs.(24) and (27), we find

$$
p_{2}\left\langle\left|\mathbf{E}_{2}\right|^{2}\right\rangle=\left(1-\frac{p_{1} d\left[d\left|\epsilon_{2}\right|^{2}-p_{2}\left|\epsilon_{1}-\epsilon_{2}\right|^{2}\right]}{\left|p_{2} \epsilon_{1}+\left(d-p_{2}\right) \epsilon_{2}\right|^{2}}\right) \mathbf{E}_{0}^{2}
$$

We should remark that although $\left|\left\langle\mathbf{E}_{1}^{2}\right\rangle\right|=\left\langle\left|\mathbf{E}_{1}\right|^{2}\right\rangle$ in MGA, $\left|\left\langle\mathbf{E}_{2}^{2}\right\rangle\right|$ is generally not equal to $\left\langle\left|\mathbf{E}_{2}\right|^{2}\right\rangle$. In fact, $\left|\left\langle\mathbf{E}_{2}^{2}\right\rangle\right| \leq\left\langle\left|\mathbf{E}_{2}\right|^{2}\right\rangle$. A common error in the literature is to treat these averages equal even when $\chi$ is complex. If we write $\epsilon_{1}=\chi_{1}\left\langle\left|\mathbf{E}_{1}\right|^{2}\right\rangle$ and $\epsilon_{2}=\chi_{2}\left\langle\left|\mathbf{E}_{2}\right|^{2}\right\rangle$, then Eqs.(27)-(28) can be solved self-consistently for $\left\langle\left|\mathbf{E}_{1}\right|^{2}\right\rangle$ and $\left\langle\left|\mathbf{E}_{2}\right|^{2}\right\rangle$ and hence the effective nonlinear response can be calculated.

In Fig.囵, we present the MGA results in three dimensions (3D). We let $\chi_{1}=1+3 i$ and $\chi_{2}=3+i$ and compute $\chi_{e}$ as a function of $p_{1}$. As $p_{1}$ increases, $\operatorname{Re}\left(\chi_{e}\right)$ decreases from $\operatorname{Re}\left(\chi_{2}\right)=3$ towards $\operatorname{Re}\left(\chi_{1}\right)=1$ while $\operatorname{Im}\left(\chi_{e}\right)$ increases from $\operatorname{Im}\left(\chi_{2}\right)=1$ towards $\operatorname{Im}\left(\chi_{1}\right)=3$. Since the contrast between the two components is relatively small, the local field averages $\left\langle\left|\mathbf{E}_{1}\right|^{2}\right\rangle$ and $\left\langle\left|\mathbf{E}_{2}\right|^{2}\right\rangle$ remain close to unity. However, unlike $\left|\left\langle\mathbf{E}_{1}^{2}\right\rangle\right|,\left|\left\langle\mathbf{E}_{2}^{2}\right\rangle\right|$ shows a significant deviation from $\left\langle\left|\mathbf{E}_{2}\right|^{2}\right\rangle$, indicating the nonuniformity of local field in the host.

For two-component composites, it has proved convenient to adopt the spectral representation of the effective linear response [3]: Let $v=1-\epsilon_{1} / \epsilon_{2}, w=1-\epsilon_{e} / \epsilon_{2}$, and $s=1 / v$, we find

$$
w(s)=\int_{0}^{1} \frac{m\left(s^{\prime}\right) \mathrm{d} s^{\prime}}{s-s^{\prime}}
$$

where $m(s)$ is the spectral density which is obtained through a limiting process:

$$
m(s)=\lim _{\eta \rightarrow 0^{+}}-\frac{1}{\pi} \operatorname{Im} w(s+i \eta) .
$$

Furthermore, $m(s)$ obeys the sum rule 


$$
\int_{0}^{1} m\left(s^{\prime}\right) \mathrm{d} s^{\prime}=p_{1}
$$

Eqs.(24)-(28) can readily be converted into the spectral representation. We find

$$
w=\frac{p_{1}}{s-s_{1}}
$$

where $s_{1}=p_{2} / d$. From Eq.(30), we obtain the spectral density in MGA:

$$
m(s)=p_{1} \delta\left(s-s_{1}\right)
$$

Similarly, the local field averages [Eqs.(25)-(28)] are given by

$$
\begin{gathered}
\left\langle\mathbf{E}_{1}^{2}\right\rangle=\frac{\mathbf{E}_{0}^{2}}{\left(1-v s_{1}\right)^{2}}, \\
\left\langle\left|\mathbf{E}_{1}\right|^{2}\right\rangle=\frac{\mathbf{E}_{0}^{2}}{\left|1-v s_{1}\right|^{2}}, \\
p_{2}\left\langle\mathbf{E}_{2}^{2}\right\rangle=\left(1-\frac{p_{1}\left(1-v^{2} s_{1}\right)}{\left(1-v s_{1}\right)^{2}}\right) \mathbf{E}_{0}^{2} \\
p_{2}\left\langle\left|\mathbf{E}_{2}\right|^{2}\right\rangle=\left(1-\frac{p_{1}\left(1-|v|^{2} s_{1}\right)}{\left|1-v s_{1}\right|^{2}}\right) \mathbf{E}_{0}^{2}
\end{gathered}
$$

If we let $v=1-\chi_{1}\left\langle\left|\mathbf{E}_{1}\right|^{2}\right\rangle / \chi_{2}\left\langle\left|\mathbf{E}_{2}\right|^{2}\right\rangle$ and solve Eqs.(35) and (37) self-consistently for $\left\langle\left|\mathbf{E}_{1}\right|^{2}\right\rangle$ and $\left\langle\left|\mathbf{E}_{2}\right|^{2}\right\rangle$, the effective nonlinear response can be determined. Although the solution appears somewhat simpler, the same results are obtained.

\section{EFFECTIVE MEDIUM APPROXIMATION}

The Bruggeman effective-medium approximation (EMA) is known to be symmetrical with respect to interchanging components 1 and 2 [5]. Note that there is a percolation threshold in the theory. For a random linear composite of $\epsilon_{1}$ and $\epsilon_{2}$ at volume fractions $p_{1}$ and $p_{2}$ respectively, the self-consistency equations reads [5]:

$$
p_{1} \frac{\epsilon_{1}-\epsilon_{e}}{\epsilon_{1}+(d-1) \epsilon_{e}}+p_{2} \frac{\epsilon_{2}-\epsilon_{e}}{\epsilon_{2}+(d-1) \epsilon_{e}}=0
$$

where $d$ is the dimensionality of composites. Solving Eq.(38), we obtain $\epsilon_{e}$ and hence $w$ and the spectral density 


$$
m(s)=\frac{d p_{1}-1}{d-1} \delta(s) \theta\left(d p_{1}-1\right)+m_{1}(s)
$$

where $\delta(s)$ and $\theta\left(d p_{1}-1\right)$ denote the Dirac delta function and the Heavyside step function respectively, and

$$
\begin{aligned}
m_{1}(s) & =\frac{d}{2 \pi(d-1) s} \sqrt{\left(s-s_{1}\right)\left(s_{2}-s\right)}, \quad s_{1}<s<s_{2} \\
& =0, \quad \text { otherwise }
\end{aligned}
$$

where $s_{1}$ and $s_{2}$ are given by

$$
\begin{aligned}
& s_{1}=\frac{1}{d}\left(1+(d-2) p_{1}-2 \sqrt{(d-1) p_{1}\left(1-p_{1}\right)}\right), \\
& s_{2}=\frac{1}{d}\left(1+(d-2) p_{1}+2 \sqrt{(d-1) p_{1}\left(1-p_{1}\right)}\right) .
\end{aligned}
$$

When $p_{1}>1 / d$, which is the percolation threshold in EMA, component 1 percolates the composite and there is a $\delta$ function contribution to $m(s)$ at $s=0$. Eqs.(34)-(37) can readily be generalized to [6]:

$$
\begin{gathered}
p_{1}\left\langle\mathbf{E}_{1}^{2}\right\rangle=\int_{0}^{1} \frac{m\left(s^{\prime}\right) \mathrm{d} s^{\prime}}{\left(1-v s^{\prime}\right)^{2}} \mathbf{E}_{0}^{2}, \\
p_{1}\left\langle\left|\mathbf{E}_{1}\right|^{2}\right\rangle=\int_{0}^{1} \frac{m\left(s^{\prime}\right) \mathrm{d} s^{\prime}}{\left|1-v s^{\prime}\right|^{2}} \mathbf{E}_{0}^{2}, \\
p_{2}\left\langle\mathbf{E}_{2}^{2}\right\rangle=\left(1-\int_{0}^{1} \frac{\left(1-v^{2} s^{\prime}\right) m\left(s^{\prime}\right) \mathrm{d} s^{\prime}}{\left(1-v s^{\prime}\right)^{2}}\right) \mathbf{E}_{0}^{2}, \\
p_{2}\left\langle\left|\mathbf{E}_{2}\right|^{2}\right\rangle=\left(1-\int_{0}^{1} \frac{\left(1-|v|^{2} s^{\prime}\right) m\left(s^{\prime}\right) \mathrm{d} s^{\prime}}{\left|1-v s^{\prime}\right|^{2}}\right) \mathbf{E}_{0}^{2} .
\end{gathered}
$$

One can check that when the MGA spectral density [Eq.(33)] is used, Eqs.(34)-(37) are recovered. Again we let $v=1-\chi_{1}\left\langle\left|\mathbf{E}_{1}\right|^{2}\right\rangle / \chi_{2}\left\langle\left|\mathbf{E}_{2}\right|^{2}\right\rangle$. Eqs.443) and (45) are coupled integral equations. They can be solved numerically for $\left\langle\left|\mathbf{E}_{1}\right|^{2}\right\rangle$ and $\left\langle\left|\mathbf{E}_{2}\right|^{2}\right\rangle$ and hence the effective nonlinear response can be determined.

In Fig.2, we present the EMA results in 3D. We let $\chi_{1}=1+3 i$ and $\chi_{2}=3+i$. Fig.2 exhibits similar behavior as Fig.1, however, with some differences. Here $\operatorname{Re}\left(\chi_{e}\right)$ and $\operatorname{Im}\left(\chi_{e}\right)$ cross at $p_{1}=0.5$ because EMA describes the symmetric microstructure and we have the 
symmetric choice of $\chi_{1}$ and $\chi_{2}$. Other quantities, like the local field averages $\left\langle\left|\mathbf{E}_{1}\right|^{2}\right\rangle,\left\langle\left|\mathbf{E}_{2}\right|^{2}\right\rangle$, and $\left|\left\langle\mathbf{E}_{1}^{2}\right\rangle\right|,\left|\left\langle\mathbf{E}_{2}^{2}\right\rangle\right|$ also cross at $p_{1}=0.5$. In EMA, both the local fields in the inclusion and host regions are not uniform.

\section{DISCUSSION AND CONCLUSION}

The model may be applicable to lossy dielectric composite materials under an intense electric, available from laser. The present formalism can naturally be extended to optical properties of nonlinear composites [0]. When the size of the inclusion is smaller than the wavelength of light, we can use the quasi-static limit. The mean-field theory can be used to calculate the optical nonlinearity.

In conclusion, we extend the mean-field theory for lossy nonlinear composites, described by complex and field-dependent dielectric functions. We developed self-consistent equations with the aid of the spectral representation of the linear composites. We examine two important types of microstructure, namely, the Maxwell-Garnett approximation and the effective medium approximation to illustrate the present theory.

\section{ACKNOWLEDGMENTS}

This work was supported by the Research Grants Council under project number CUHK 461/95P. I acknowledge useful conversation with Professor Ping Sheng. 


\section{REFERENCES}

[1] K. W. Yu, P. M. Hui and H. C. Lee, Phys. Lett. A 210, 115 (1996); see also D. J. Bergman, Phys. Rep. 9, 378 (1978).

[2] D. Stroud and V. E. Wood, J. Opt. Soc. Am. B 6, 778 (1989).

[3] D. J. Bergman and D. Stroud, in: Solid State Physics, Vol. 46, edited by H. Ehrenreich and D. Turnbull (Academic Press, New York, 1992), p.147; G. W. Milton, J. Appl. Phys. 52, 5286 (1980); G. W. Milton, Appl. Phys. A 26, 1207 (1981).

[4] J. C. Maxwell-Garnett, Philos. Trans. R. Soc. 203, 385 (1904); 205, 237 (1906).

[5] D. A. G. Bruggeman, Ann. Phys. (Leipzig) 24, 636 (1935).

[6] Ping Sheng, private communications. These formulas can readily be verified from the formal properties of the spectral representation (D. J. Bergman and D. Stroud, in Ref. [3] ).

[7] See the articles in J. Opt. Soc. Am. B 6, (1989), special issue on Nonlinear-Optical Properties of Materials, edited by C. M. Bowden and J. W. Haus. 


\section{FIGURES}

FIG. 1. Real and imaginary parts of the effective nonlinear response plotted against volume fraction of the inclusion in the MGA with $\chi_{1}=1+3 i$ and $\chi_{2}=3+i$.

FIG. 2. Real and imaginary parts of the effective nonlinear response plotted against volume fraction of the inclusion in the EMA with $\chi_{1}=1+3 i$ and $\chi_{2}=3+i$. 
MGA

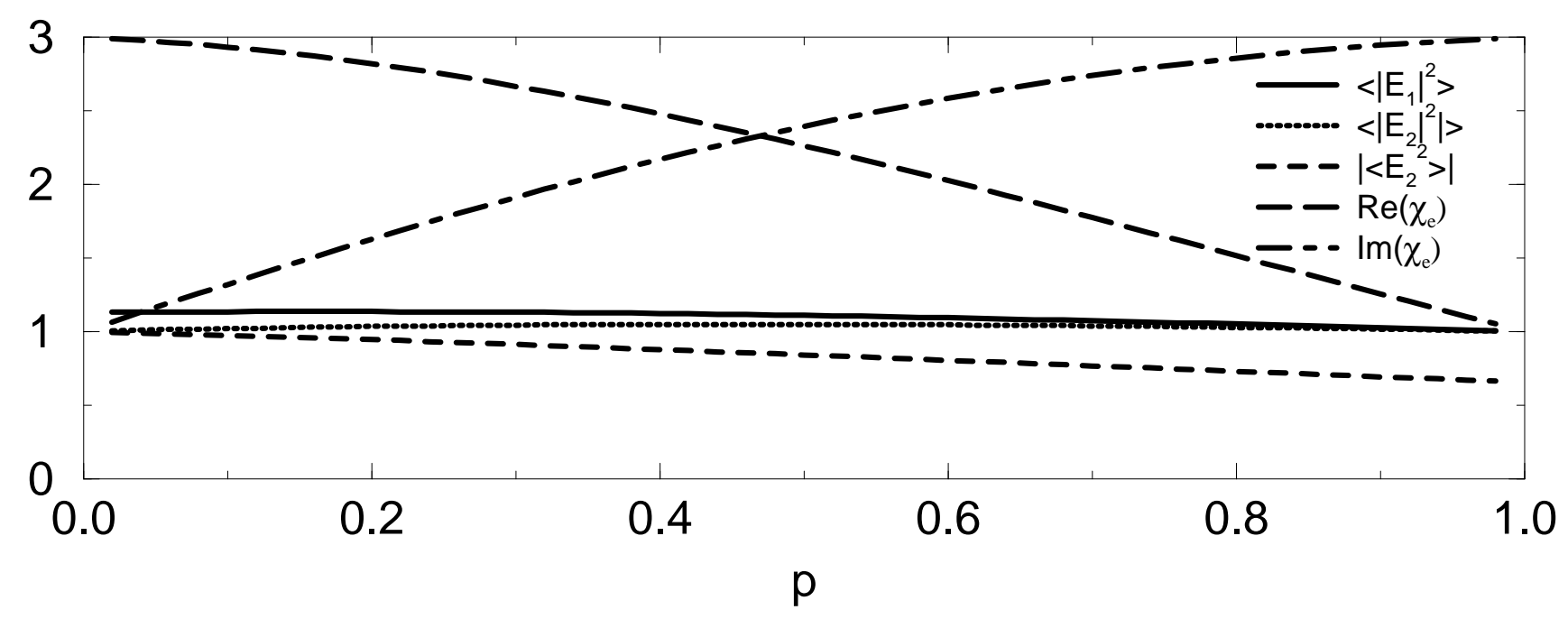

EMA

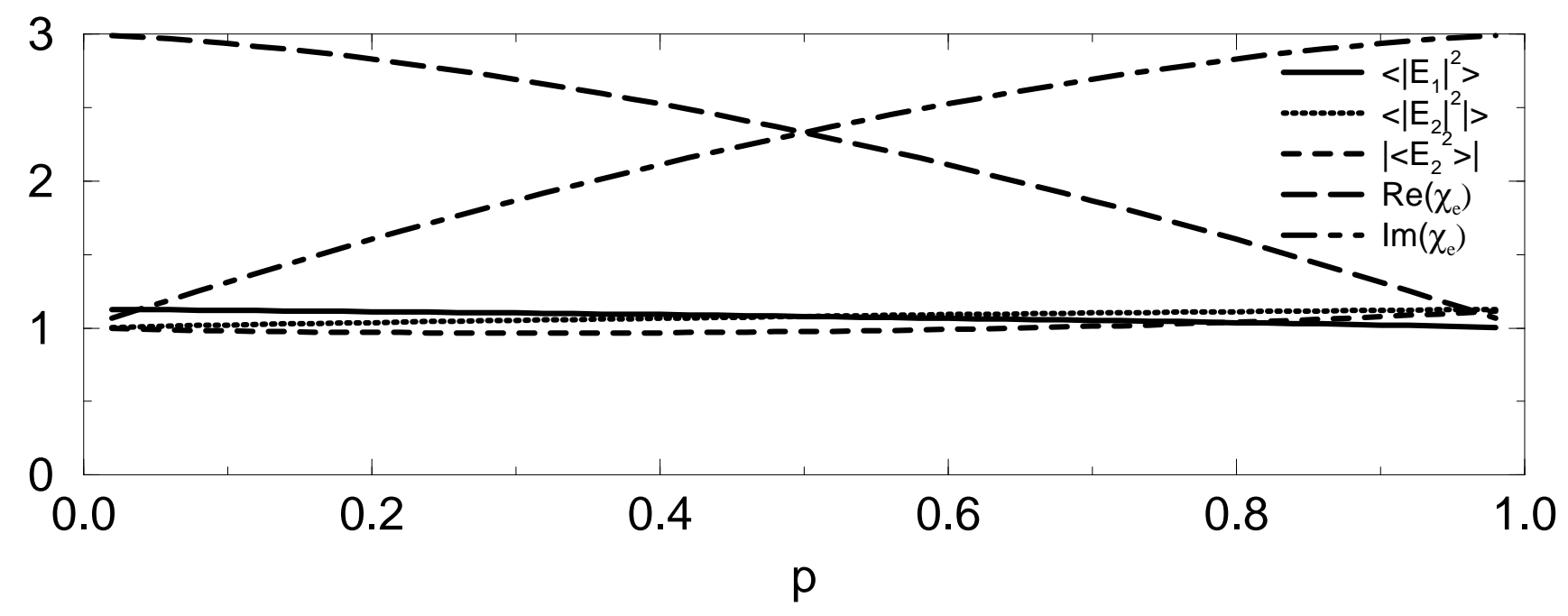

